\title{
Cross-walk of the Assessment of Spondyloarthritis International Society Health Index and Ankylosing Spondylitis Quality of Life Scores in Ankylosing Spondylitis and Non-radiographic Axial Spondyloarthritis Patients
}

\author{
James Pike $\cdot$ Yan Dong $\cdot$ James Piercy $\cdot$ Nicola Booth (D) \\ Elizabeth Holdsworth · Theresa Hunter
}

Received: February 1, 2021 / Accepted: April 2, 2021 / Published online: April 17, 2021

(C) The Author(s) 2021

\section{ABSTRACT}

Introduction: Axial spondyloarthritis (axSpA) is a chronic rheumatic disease affecting the spine and sacroiliac joints, encompassing both ankylosing spondylitis (AS) and non-radiographic axial spondyloarthritis (nr-axSpA) patients. Patient quality of life (QoL) is assessed using the Ankylosing Spondylitis Quality of Life (ASQoL) (disease-specific measure) and the Assessment of SpondyloArthritis International Society Health Index (ASAS HI) (disease-specific measure). Both ASQoL and ASAS HI have similar parameters and scoring ranges, however, their performance relative to each other is unknown. We conducted a cross-walk analysis of the ASAS $\mathrm{HI}$ to the ASQoL in AS and nr-axSpA patients. Methods: A cross-sectional survey using the Adelphi axSpA Disease Specific Programme ${ }^{\mathrm{TM}}$, conducted with rheumatologists and their consulting AS and nr-axSpA patients in the United States, was undertaken between Jun and Aug 2018. Rheumatologists provided confirmed diagnoses of AS and nr-axSpA alongside patients' demographic and clinical characteristics. Patients reported quality-of-life measures

J. Pike $\cdot$ J. Piercy $\cdot$ N. Booth $(\bowtie) \cdot$ E. Holdsworth

Adelphi Real World, Bollington, UK

e-mail: nicola.booth@adelphigroup.com

Y. Dong · T. Hunter

Eli Lilly and Company, Indianapolis, IN, USA using the validated ASAS HI and ASQoL questionnaires. Model performance was assessed by comparing root-mean squared error (RMSE) from tenfold cross-validation to determine the best mapping from ASAS HI to ASQoL, and vice versa. RMSE was calculated overall, and for lower, middle and upper thirds of the predicted scale.

Results: Data from 283 AS and 274 nr-axSpA patients were analyzed. Predicting ASAS HI using ASQoL values, the best model was nonparametric local linear regression, with overall RMSE of 1.851. Predicting ASQoL using ASAS HI values, the best model also used non-parametric local-linear regression, with overall RMSE of 2.254. In predicting ASAS HI and ASQoL, models performed better in predicting lower values in the predicted scale (RMSE 1.597, 1.871, 2.871 across thirds for ASAS HI; and 1.719, 2.577, 3.140 for ASQoL).

Conclusions: Results present a scoring algorithm for cross-walking the ASAS HI to the ASQoL and vice versa, with the approach enabling comparisons to be made across studies.

Keywords: Ankylosing spondylitis; ASAS HI; ASQoL; Cross-walk analysis; Non-radiographic axial spondyloarthritis; Patient-reported outcomes 


\section{Key Summary Points}

Patients with ankylosing spondylitis and non-radiographic axial spondyloarthritis report a similar range of symptoms, which are assessed using the Assessment of SpondyloArthritis International Society Health Index (ASAS HI) and Ankylosing Spondylitis Quality of Life (ASQoL) tools.

While these patient-reported outcome tools assess a similar range of measures, it is not known how they perform in comparison to each other across the different disease groups and if they can be used to predict one another in the absence of one.

We developed a cross-walk scoring algorithm approach using non-parametric local-linear regression to allow for comparisons to be made across different studies using ASQoL or ASAS HI to assess patient-reported outcomes.

The results of our study demonstrate a scoring algorithm for cross-walking the ASAS HI to the ASQoL and vice versa, which allows for comparisons across studies using either ASAS HI or ASQoL to be made.

\section{DIGITAL FEATURES}

This article is published with digital features, including a summary slide, to facilitate understanding of the article. To view digital features for this article go to https://doi.org/10.6084/ m9.figshare.14339597.

\section{INTRODUCTION}

Axial spondyloarthritis (axSpA) is a chronic inflammatory rheumatic disease that predominantly affects the spine and sacroiliac joints and encompasses patients with ankylosing spondylitis (AS) and non-radiographic axial spondyloarthritis (nr-axSpA) [1, 2]. Both AS and nr-axSpA patients present with similar symptoms, such as back pain, morning stiffness, reduced mobility, fatigue, difficulty sleeping, impaired social activity, and work productivity, all resulting in decreased health-related quality of life (HRQoL) [3, 4]. Clinical manifestations and disease activity measures of early AS and nraxSpA are comparable, indicating that both are part of the same disease spectrum [5]. Although not all patients with nr-axSpA progress to modified New York criteria-defined AS, the disease activity, functional impairment, HRQoL, and health status have been reported to be similar between patients with nr-axSpA and AS [3, 6-8].

HRQoL of AS and nr-axSpA patients has been assessed in previous studies using patient-reported outcome (PRO) measures such as the 36-Item Short Form Health Survey (SF-36) (general HRQoL) [9, 10], Ankylosing Spondylitis Quality of Life (ASQoL) (disease-specific measure) [11], and Assessment of SpondyloArthritis International Society Health Index (ASAS HI) (disease-specific measure) [12]. These measures were developed in order to assess HRQoL and impact of the disease on daily activities and/or social participation from a patient's perspective, as well as to quantify the relative impact of therapeutic response to treatment [11-13]. These factors are not always adequately assessed by traditional physician-focused disease-specific questionnaires as physicians are more likely to focus on measurable aspects of care such as pain, disability, and level of impairment [14].

The ASAS HI and the ASQoL quantify patient-reported HRQoL using similar parameters have a similar scoring range and have been validated in their respective patient cohorts [11-15]. However, their performance relative to each other is unknown. Since the ASQoL and ASAS HI are both used in clinical trials and realworld evidence studies of axSpA patients, it is important to be able to compare the results across studies using these measures and to assess equivalency of outcomes. Therefore, the objective of this study was to conduct a cross-walk analysis of the ASAS HI to ASQoL, and vice 
versa, in patients with physician-confirmed AS and nr-axSpA.

\section{METHODS}

\section{Study Design}

Data were used from the Adelphi axSpA Disease Specific Programme (DSP ${ }^{\mathrm{TM}}$ ) surveys collected in the United States (US) between June and August 2018 from rheumatologists and their consulting AS and nr-axSpA patients using a combination of rheumatologist-completed patient record forms and voluntary patient selfcompleted forms. DSPs are large, retrospective, point-in-time surveys; the full methodology has been described and published previously $[16,17]$.

Rheumatologists who saw $\geq 10$ axSpA patients in a typical month were eligible to participate in the study. Eligible rheumatologists completed patient record forms for their next ten consecutive patients (five AS and five nr-axSpA), with a rheumatologist-confirmed diagnosis of AS or nr-axSpA. Rheumatologists reported information on patient demographic characteristics (age, sex, body mass index [BMI], employment status, and ethnicity), disease status (severity, time since diagnosis) and current treatment patterns. Patients with a rheumatologist-confirmed diagnosis of AS and nr-axSpA were invited to complete a self-completed form independent of their rheumatologists, in which they completed patient-reported outcomes including the ASQoL and ASAS HI.

\section{Ethics}

Ethical approval was sought and granted through Western Institutional Review Board (WIRB-Study ID number 1183030). The noninterventional, observational nature of the data collection did not result in patients being placed at risk from the study. Physicians and patients provided informed consent to participate in the study and did not provide any personally identifiable information. All responses were anonymized to preserve respondent (physician and patient) confidentiality and all participating physicians and patients were assigned a study number to aid anonymous data collection and allow linkage of data during data collection and analysis, as well as other regional guidelines and regulations where appropriate.

\section{Outcome Measures}

\section{ASQoL}

The ASQoL questionnaire was developed in 2003 and is a self-administered 18-item questionnaire around the needs-based QoL model. The ASQoL has been utilized in both clinical research and daily practice to assess patient-reported HRQoL [11]. It is well accepted by AS patients, taking about 4 min to complete, and is a valuable tool for assessing the impact of interventions for AS and evaluating models of service delivery, covering functional, emotional, pain and fatigue domains, and the impact of AS on a patient's HRQoL [18]. Patients are asked to respond "Yes" (equaling a score of 1) or "No" (equaling a score of 0 ) to each statement based on how they feel at the time of completion. All item scores are summed to give a total score ranging from 0 (good HRQoL) to 18 (poor HRQoL) [19]. A total score cannot be calculated if more than three items (i.e., $20 \%$ of the data) are missing. Published research has indicated that differences of 1-2 points in ASQoL scores are clinically significant [20].

\section{ASAS HI}

The ASAS HI is a new health index tool based on the International Classification of Functioning, Disability and Health (ICF) core set developed specifically for patients with axSpA to measure the concepts of functioning, disability, and health [12]. The ASAS HI is a linear composite questionnaire covering 17 categories, forming a unidimensional scale providing a sum score representing a wide spectrum of different levels of functioning, including pain, function, sleep, mobility, self-care, and social interactions [21]. Patients are asked to respond "Yes" or "No" to a list of statements based on how they feel at the time of completion. All statements have an equal value, with "Yes" equaling a score of 1 and 
"No" equaling a score of 0 . All item scores are summed to give a total score ranging from 0 (good HRQoL) to 17 (poor HRQoL). Items No 7 (I have lost interest in sex) and 8 (I have difficulty operating the pedals in my car) are not applicable to all patients. For those patients who ticked the response "not applicable", the sum score is analyzed based on $n=16$ or $n=15$, respectively. An overall score is calculated, with a lower score representing better functioning and higher HRQoL [12]. A total score cannot be calculated if more than three items (i.e., $20 \%$ of the data) are missing. The ASAS HI has been validated in a cross-sectional international observational study in patients with AS, nraxSpA, and peripheral SpA (pSpA) across 23 countries [15]. Reliability in patients with stable disease who had repeat measurements was good; values $\leq 5.0$ distinguish good health, as opposed to moderate health, with values $\geq 12.0$ representing poor health. The smallest detectable change in the measure has been reported to be 3 points $[12,15]$.

\section{Statistical Analysis}

\section{Patients Demographic and Clinical Characteristics}

Patient demographic and clinical characteristics will be reported among three groups, AS, nraxSpA, and overall patients. The continuous variable will be reported with number of patients, mean, standard deviation, minimum, and maximum. Count and percentage will be presented in each specific group for categorical variable. Base sizes can vary between characteristics due to missing values in physician completed patient record forms.

\section{Mapping Between ASAS HI and ASQoL Using Cross-validation}

$K$-fold cross-validation is a technique in which patients in the dataset are randomly partitioned into $k$ sub-samples [22]. One sub-sample is set aside as the validation data for testing the predictive model and the remaining $k-1$ subsamples are used to generate the predictive model. The cross-validation process is then repeated $k-1$ more times, with each of the $k$ sub-samples used exactly once as validation data. Model accuracy measures are calculated on the validation data. These measures are considered "out of sample" as they are calculated on data not used in the predictive model.

Tenfold cross validation was used to determine the best models in mapping between ASAS $\mathrm{HI}$ and ASQoL [23]. Mapping was conducted from ASAS HI to ASQoL and also from ASQoL to ASAS HI.

A range of models were assessed. The mean score was first generated, this served as a benchmark for other models. If models could not perform better than assigning the mean score to the data, then they were considered poor models.

Regression models were generated, utilizing ordinary least squares and generalized linear models [24]. For generalized linear models, Gaussian, Poisson, negative binomial, gamma, and inverse Gaussian families were used, with identity, log, and power (from -1 to 1.2 in increments of 0.05) links utilized.

Fractional logit was also performed [25], conducted on scores rescaled to $[0,1]$ interval, utilizing robust standard errors.

Nonparametric local-linear regression using the Epanechnikov kernel was also undertaken [26]. Like linear regression, nonparametric regression models the mean of the outcome conditional on the covariates, but unlike linear regression, it makes no assumptions about the functional form of the relationship between the outcome and the covariates. Nonparametric local-linear regression may be used to model the mean of a continuous, count, or binary outcome.

In the models described above, covariates were also utilized, including age, sex, BMI, employment status, ethnicity, disease severity, and treatment. Splines of continuous predictors/covariates were also investigated, including linear splines and restricted cubic splines, with knots derived from percentiles of independent variables. Interaction terms between predictors/covariates were also investigated.

All models had their performance assessed by utilizing root-mean squared error (RMSE) as an accuracy measure. The RMSE is the square root of the variance of the residuals and can be 
interpreted as the standard deviation of the unexplained variance [27]. Therefore, lower values of RMSE indicate better fit, and well-fitting models have an RMSE which is lower than the clinically meaningful difference in the ASAS HI or ASQoL. Additionally, the RMSE was examined at the lower, middle, and upper thirds of the predicted scale. This allowed us to determine if the model fitted well over the full range of values.

The model with the lowest RMSE in crossvalidation was deemed the best. However, an alternative model would have been selected if the RMSE values indicated that the model performed particularly poorly in certain ranges of the scale. For the selected model, appropriate summary output statistics and regression tables are given. A summary of how values map from one scale to the other and scatter plots of recorded values against predicted values are also presented.

Conventionally, in this study alpha levels of 0.05 were deemed significant. However, the methods used were not driven by significance testing. Additionally, no adjustments for multiplicity were made. Analysis was undertaken in Stata 16.1 (StataCorp. 2019. Stata Statistical Software: Release 16. College Station, TX, USA).

\section{RESULTS}

\section{Demographics}

In total, 63 rheumatologists completed PRFs and participated in this survey, with data from 283 AS patient and $274 \mathrm{nr}$-axSpA patient PSCs included in the cross-walk analysis. Patient demographic and clinical characteristics of the overall patient cohort $(n=557)$, and of the two patient sub-groups, are shown in Table 1 . The mean age of the cohort was 45.3 years, and the mean time since diagnosis was 5.1 years. In total, $60.7 \%$ of the cohort were male, $82.2 \%$ were Caucasian/white, and the mean BMI was 27.3. Overall, $64.1 \%$ were categorized with mild disease, $32.5 \%$ moderate disease, and 3.4\% having severe disease. The full-time employment rate was $68.6 \%$.

\section{Description of ASQoL and ASAS HI Scores}

The distribution of ASQoL and ASAS HI scores across both patient groups is shown in Fig. 1. There was no significant difference in the distribution of scores. Overall, the mean \pm standard deviation (SD) overall ASQoL score was $6 \pm 5.4$, AS patients had a mean score of $6.1 \pm 5.4$, and nr-axSpA patients had a mean score of $5.8 \pm 5.3 \quad(p=0.5)$. The mean \pm SD ASAS HI score across both patient populations was $5.5 \pm 4.2$, patients with AS had a mean score of $5.7 \pm 4.4$, with nr-axSpA patients having a mean score of $5.3 \pm 4.1(p=0.23)$.

\section{Regression Analysis}

Over 600 regression models were tested for predicting ASQoL and ASAS HI. Table 2 gives the results of this analysis. Models both with and without covariates had superior performance when predicting lower values and performed worst when predicting higher values (RMSE 1.597, 1.871, 2.871 across thirds for ASAS HI without covariates; and 1.719, 2.577, 3.140 for ASQoL without covariates). Overall, lower and middle RMSE values for models with and without covariates were lower than clinically significant differences for ASQoL and ASAS HI, as described above. Model predictions are shown in Figs. 2 and 3 for predicting ASQoL and ASAS HI, respectively, indicating the line of best fit between the two parameters based on the performance of each model with and without covariates. Figures 2 and 3 also include observed values and the $95 \%$ confidence intervals of predicted values. Full regression tables for the best-performing models can also be found in the appendix.

\section{DISCUSSION}

The ASQoL and ASAS HI are both used in clinical trials and real-world evidence studies of axSpA patients. While many of the questions asked by both the ASQoL and ASAS are comparable, and the two scales have a similar scoring range, it is important to be able to compare 
Table 1 Patient demographics

\begin{tabular}{|c|c|c|c|c|}
\hline & $\begin{array}{l}\text { Overall } \\
n=557\end{array}$ & $\begin{array}{l}\text { AS } \\
n=283\end{array}$ & $\begin{array}{l}\text { nr-axSpA } \\
n=274\end{array}$ & $p$ value \\
\hline \multicolumn{5}{|l|}{ Age } \\
\hline$n$ & 557 & 283 & 274 & 0.001 \\
\hline Mean & 45.3 & 47.1 & 43.4 & $(\mathrm{TT})$ \\
\hline SD & 13.7 & 14.3 & 12.7 & \\
\hline Median & 44 & 46 & 41 & \\
\hline IQR & $35.0,55.0$ & $37.0,57.0$ & $34.0,52.0$ & \\
\hline Min, $\max$ & $18.0,84.0$ & $19.0,84.0$ & $18.0,81.0$ & \\
\hline \multicolumn{5}{|l|}{ Years since diagnosis } \\
\hline$n$ & 467 & 224 & 243 & $<0.0001$ \\
\hline Mean & 5.1 & 6.7 & 3.7 & $(\mathrm{TT})$ \\
\hline SD & 6.9 & 8.5 & 4.7 & \\
\hline Median & 2.8 & 3.7 & 2.1 & \\
\hline IQR & $1.3,6.2$ & $1.6,8.0$ & $1.1,4.7$ & \\
\hline Min, $\max$ & $0.0,67.6$ & $0.0,67.6$ & $0.0,36.2$ & \\
\hline \multicolumn{5}{|l|}{ Sex, $n(\%)$} \\
\hline$n$ & 557 & 283 & 274 & $<0.0001$ \\
\hline Male & $338(60.7)$ & $195(68.9)$ & $143(52.2)$ & (FE) \\
\hline Female & $219(39.3)$ & $88(31.1)$ & $131(47.8)$ & \\
\hline \multicolumn{5}{|l|}{ BMI } \\
\hline$n$ & 557 & 283 & 274 & 0.3333 \\
\hline Mean & 27.3 & 27.5 & 27.1 & $(\mathrm{TT})$ \\
\hline SD & 4.7 & 4.7 & 4.8 & \\
\hline Median & 26.6 & 26.6 & 26.3 & \\
\hline IQR & $24.3,29.4$ & $24.6,29.5$ & $23.7,29.4$ & \\
\hline Min, $\max$ & $16.2,51.7$ & $16.2,51.7$ & $17.5,44.3$ & \\
\hline \multicolumn{5}{|l|}{ Employment status, $n$ (\%) } \\
\hline$n$ & 557 & 283 & 274 & 0.0209 \\
\hline Working full-time & $382(68.6)$ & $193(68.2)$ & $189(69.0)$ & $(\mathrm{CH})$ \\
\hline Working part-time & $38(6.8)$ & $17(6.0)$ & $21(7.7)$ & \\
\hline On long-term sick leave & $8(1.4)$ & $6(2.1)$ & $2(0.7)$ & \\
\hline Homemaker & $36(6.5)$ & $12(4.2)$ & $24(8.8)$ & \\
\hline Student & $18(3.2)$ & $7(2.5)$ & $11(4.0)$ & \\
\hline Retired & $45(8.1)$ & $30(10.6)$ & $15(5.5)$ & \\
\hline
\end{tabular}


Table 1 continued

\begin{tabular}{|c|c|c|c|c|}
\hline & $\begin{array}{l}\text { Overall } \\
n=557\end{array}$ & $\begin{array}{l}\text { AS } \\
n=283\end{array}$ & $\begin{array}{l}\text { nr-axSpA } \\
n=274\end{array}$ & $p$ value \\
\hline Unemployed & $28(5.0)$ & $18(6.4)$ & $10(3.6)$ & \\
\hline Don't know & $2(0.4)$ & $0(0.0)$ & $2(0.7)$ & \\
\hline \multicolumn{5}{|l|}{ Ethnicity, $n(\%)$} \\
\hline$n$ & 557 & 283 & 274 & 0.5065 \\
\hline White/Caucasian & $458(82.2)$ & $236(83.4)$ & $222(81.0)$ & (FE) \\
\hline Non-white/Caucasian & $99(17.8)$ & $47(16.6)$ & $52(19.0)$ & \\
\hline \multicolumn{5}{|l|}{ Current severity, $n(\%)^{+}$} \\
\hline$n$ & 557 & 283 & 274 & $<0.0001$ \\
\hline Mild & $357(64.1)$ & $170(60.1)$ & $187(68.2)$ & $(\mathrm{CH})$ \\
\hline Moderate & $181(32.5)$ & $94(33.2)$ & $87(31.8)$ & \\
\hline Severe & $19(3.4)$ & $19(6.7)$ & $0(0.0)$ & \\
\hline \multicolumn{5}{|l|}{ Medication use, $n(\%)$} \\
\hline$n$ & 557 & 283 & 274 & 0.003 \\
\hline No biologic & $166(29.8)$ & $68(24.0)$ & $98(35.8)$ & $(\mathrm{FE})$ \\
\hline Biologic & $391(70.2)$ & $215(76.0)$ & $176(64.2)$ & \\
\hline \multicolumn{5}{|c|}{ Number of concomitant conditions } \\
\hline$n$ & 550 & 280 & 270 & 0.1318 \\
\hline Mean & 1.2 & 1.3 & 1.1 & $(\mathrm{TT})$ \\
\hline SD & 1.4 & 1.6 & 1.2 & \\
\hline Median & 1 & 1 & 1 & \\
\hline IQR & $0.0,2.0$ & $0.0,2.0$ & $0.0,2.0$ & \\
\hline Min, $\max$ & $0.0,10.0$ & $0.0,10.0$ & $0.0,6.0$ & \\
\hline \multicolumn{5}{|c|}{ Charlson comorbidity index } \\
\hline$n$ & 550 & 280 & 270 & 0.6291 \\
\hline Mean & 0.1 & 0.1 & 0.1 & (TT) \\
\hline SD & 0.4 & 0.5 & 0.4 & \\
\hline Median & 0 & 0 & 0 & \\
\hline $\mathrm{IQR}$ & $0.0,0.0$ & $0.0,0.0$ & $0.0,0.0$ & \\
\hline Min, $\max$ & $0.0,4.0$ & $0.0,4.0$ & $0.0,4.0$ & \\
\hline
\end{tabular}

Base sizes can vary between characteristics, due to missing values in physician completed patient record forms $A S$ ankylosing spondylitis, $C H$ Chi-squared test, $F E$ Fisher's exact test, IQR interquartile range, $n r$-axSpA non-radiographic axial spondyloarthritis, $S D$ standard deviation, $T T t$ test

+ Physician-reported outcome 


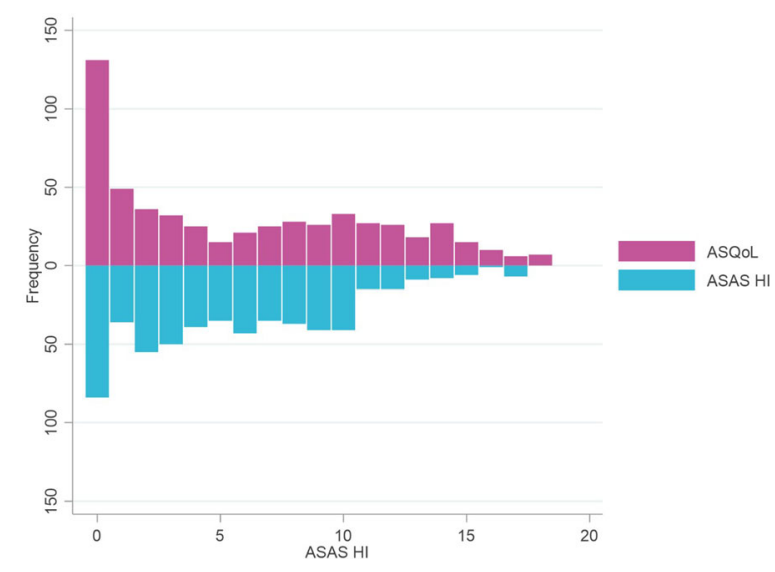

Fig. 1 Distribution of ASQoL and ASAS HI scores. The distribution of ASQoL and ASAS HI scores reported across both patient groups. ASAS HI Assessment of SpondyloArthritis International Society Health Index, ASQoL Ankylosing Spondylitis Quality of Life the results across studies using these measures and to assess equivalency of outcomes. This is the first study demonstrating a cross-walk scoring algorithm for the ASQoL to the ASAS HI, and vice versa. The ASAS-HI tool was first released in 2014, and thus the published evidenced is lower than for the ASQoL tool which has been in use since 2003. This cross-walk approach is of value, in that it facilitates comparisons of patient-reported outcomes relating to disease severity and treatment response between different studies published over time, conducted in different patient groups using either the ASQoL or ASAS-HI scales, thereby increasing transparency and reducing uncertainty surrounding decision-making processes.

In a previous study of 140 axSpA patients, ASAS HI scores were obtained and compared alongside other disease outcome activity measures, with a correlation coefficient of 0.784

Table 2 Regression model cross-validation results

\begin{tabular}{|c|c|c|c|c|c|c|}
\hline Model & Description & Covariates & $\begin{array}{l}\text { Overall } \\
\text { RMSE }\end{array}$ & $\begin{array}{l}\text { Lower } \\
\text { RMSE }\end{array}$ & $\begin{array}{l}\text { Middle } \\
\text { RMSE }\end{array}$ & $\begin{array}{l}\text { Upper } \\
\text { RMSE }\end{array}$ \\
\hline \multicolumn{7}{|c|}{ Predicting ASAS HI } \\
\hline Mean & Constant model & Constant only & 4.246 & 3.789 & 3.199 & 8.438 \\
\hline $\begin{array}{l}\text { Without } \\
\text { covariates }\end{array}$ & $\begin{array}{l}\text { Nonparametric } \\
\text { local-linear } \\
\text { regression }\end{array}$ & ASQoL & 1.851 & 1.597 & 1.871 & 2.871 \\
\hline $\begin{array}{l}\text { With } \\
\text { covariates }\end{array}$ & $\begin{array}{l}\text { GLM with gaussian } \\
\text { family and power } \\
\text { link }(\wedge 1.15)\end{array}$ & $\begin{array}{l}\text { Linear splines of ASQoL, age, employment } \\
\text { status, age/employment status interaction }\end{array}$ & 1.807 & 1.602 & 1.755 & 2.851 \\
\hline \multicolumn{7}{|c|}{ Predicting ASQoL } \\
\hline Mean & Constant model & Constant only & 5.389 & 4.714 & 3.947 & 8.966 \\
\hline $\begin{array}{l}\text { Without } \\
\text { covariates }\end{array}$ & $\begin{array}{l}\text { Nonparametric } \\
\text { local-linear } \\
\text { regression }\end{array}$ & ASAS HI & 2.254 & 1.719 & 2.577 & 3.140 \\
\hline $\begin{array}{l}\text { With } \\
\text { covariates }\end{array}$ & $\begin{array}{l}\text { GLM with Gaussian } \\
\text { family and power } \\
\text { link }\left({ }^{\wedge} 0.55\right)\end{array}$ & $\begin{array}{l}\text { Linear splines of ASQoL, age, sex, BMI, } \\
\text { ethnicity, disease severity, male/disease } \\
\text { severity interaction, male/ethnicity } \\
\text { interaction, sex/BMI interaction }\end{array}$ & 2.173 & 1.626 & 2.509 & 3.048 \\
\hline
\end{tabular}

ASAS HI Assessment of SpondyloArthritis International Society Health Index, ASQoL Ankylosing Spondylitis Quality of Life, $B M I$ body mass index, GLM generalized linear model, RMSE root mean square error 

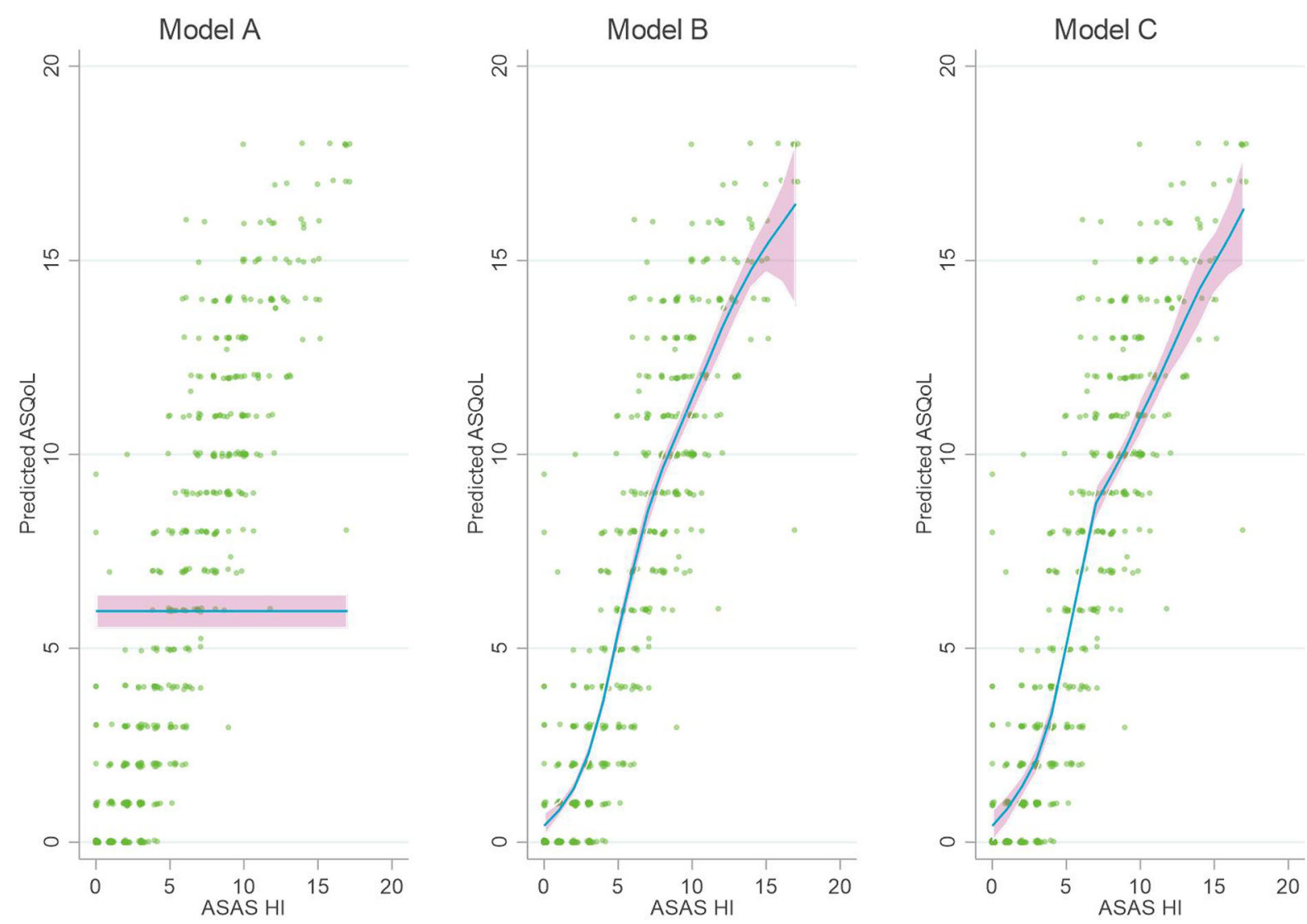

Fig. 2 Models for predicting ASQoL scores from ASAS HI scores. Models for predicting ASQoL scores from ASAS HI scores, indicating the line of best fit between the two parameters based on the performance of each model. Observed values shown in green and 95\% confidence intervals of predicted values are shown in pink. a Mean

observed between ASAS HI and ASQoL [28]. In addition, a systematic review of various PRO measures for SpA identified a wide range of QoL domains of relevance, with minimal differences observed between patients with axial and peripheral SpA [29]. The authors concluded that peripheral SpA PROs can be used to measure QoL of patients with axial SpA. However, our study builds on both of these by mapping the two domains directly against each other in the same patient population, thereby creating a mathematical model in order to better understand the relationship between the two scoring systems.

We found that when predicting ASAS HI scores using ASQoL values, the best model was model. b Non-parametric local-linear regression, without covariates. c GLM with Gaussian family and power link $\left({ }^{\wedge} 0.55\right)$, with covariates. ASAS HI Assessment of SpondyloArthritis International Society Health Index, ASQoL Ankylosing Spondylitis Quality of Life

non-parametric local-linear regression, with an overall RMSE of 1.851. When predicting ASQoL scores using ASAS HI values, similarly to the opposite direction cross-walk, the best model was also non-parametric local-linear regression, with an overall RMSE of 2.254. The RMSE is the square root of the variance of the residuals and can be interpreted as the standard deviation of the unexplained variance, with lower values representing a better fit. In this study, the RMSE observed in both best fit models was less than the minimally observed clinical difference for both scales (two for ASQoL and three for ASAS $\mathrm{HI})$, indicating that outcomes of our analyses can be considered well-fitting models. 

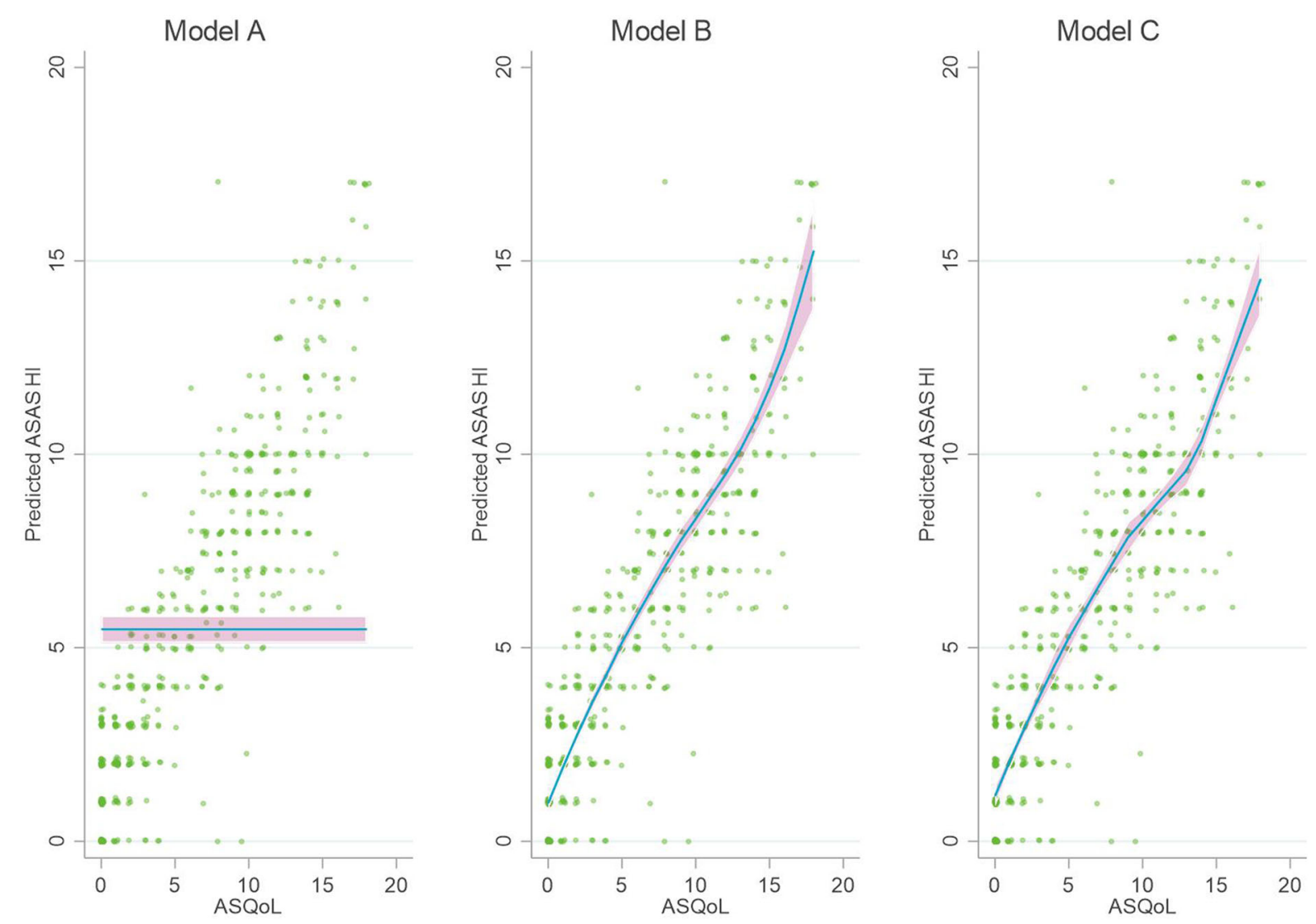

Fig. 3 Models for predicting ASAS HI scores from ASQoL scores. Models for predicting ASAS HI scores from ASQoL scores, indicating the line of best fit between the two parameters based on the performance of each model. Observed values shown in green and 95\% confidence intervals of predicted values are shown in pink.

In predicting ASQoL and ASAS HI, our models performed better in predicting lower values in the predicted scale. One explanation for this was that because there were more patients at the lower end of the distribution, there were more comparable data points to match. Patients with more severe disease had a greater range of potential disease variables, therefore the crosswalk matching between the two scales may not have been as precise.

There was no significant difference in the distribution of ASQOL and ASAS HI scores across both patient groups. The mean overall ASQoL score was $6 \pm 5.4$, and the mean overall ASAS HI score was $5.5 \pm 4.2$, which corresponds with the trends and distributions seen in previously a Mean model. b Non-parametric local-linear regression, without covariates. c GLM with Gaussian family and power link (^1.15), with covariates. ASAS HI Assessment of SpondyloArthritis International Society Health Index, ASQoL Ankylosing Spondylitis Quality of Life

published literature. In the original study detailing the development of the ASQoL tool [11], the UK subset $(n=210)$ had a mean $9.5 \pm 5.3$ at time 1 and mean $8.8 \pm 5.7$ at time 2 , and the Dutch subset $(n=158)$ had a mean $6.7 \pm 4.8$ at time 1 , and mean $6.2 \pm 4.8$ at time 2 . In the global study validating the ASAS HI undertaken in 23 different countries ( $n=1548$ patients), the mean score was $6.7 \pm 4.3$ [15]. Taken together, these indicate that the population used in this study was representative and comparable with wider patient demographic.

In our study, some differences in clinical and demographic characteristics were observed between AS and nr-axSpA patients. For example, AS patients were older, had been diagnosed 
longer, were more likely to be retired and male, and had a higher disease severity than nr-axSpA patients. However, all of these findings were to be expected; AS is a more progressed condition than nr-axSpA and these differences are well described in the existing scientific literature $[6,30]$. In any case, these differences observed between the AS and the nr-axSpA patients do not influence the outcome of the crosswalk methodology, as all patients completed both PRO tools. The overall distribution of scores for each instrument was skewed towards higher values for AS patients, but this simply reflects the higher disease severity. Including both types of patients strengthens the robustness and the reliability of the analysis, since we are able to analyze scores both at the upper end and lower end of each scale.

\section{Strengths and Limitations}

One of the key strengths of the data set used is that patients were enrolled by their treating rheumatologist, so only patients with a confirmed diagnosis of AS or nr-axSpA could be included. As mentioned above, patients presented with all degrees of severity, leading to a distribution of scores for both instruments across the whole range of values. This proposed relationship is strongly driven by the collected data, however there may be rare instances where this may not hold true. A general limitation, one in common with all real-world research, is that analysis is restricted only to those patients who are willing to participate. However, since it is the same patients completing both instruments, the study is internally consistent; as the analysis includes patients from all severity levels, the patients are representative of the whole AS and nr-axSpA population. One other limitation that needs to be mentioned is that the study included patients only from the US. If the results were to be applied to other populations, then some caution might be needed with interpretation if it is likely that an ex-US patient might score one of the instruments differently.

\section{CONCLUSIONS}

The results of this study demonstrate a scoring algorithm for cross-walking the ASAS HI to the ASQoL and vice versa, with the approach allowing for comparisons across studies using either ASAS HI or ASQoL to be made. Future research is needed to confirm our findings and the clinical usefulness of this study.

\section{ACKNOWLEDGEMENTS}

Funding. Data collection was undertaken by Adelphi Real World as part of an independent survey. The study described here using these data, and the associated Rapid Service Fee were funded by Eli Lilly and Co., Indianapolis, USA, who did not influence the original survey through either contribution to the design of questionnaires or data collection.

Authorship. All named authors meet the International Committee of Medical Journal Editors (ICMJE) criteria for authorship of this article, take responsibility for the integrity of the work as a whole, and have given their approval for this version to be published.

James Pike: Design, analysis, and interpretation of data for the manuscript. Drafted and critically revised the manuscript for intellectual content.

Yan Dong: Conception, design, analysis, and interpretation of data for the manuscript and critically revised the manuscript for intellectual content.

James Piercy: Conception, design, and interpretation of data for the manuscript and critically revised the manuscript for intellectual content.

Nicola Booth: Acquisition and interpretation of data for the manuscript and critically revised the manuscript for intellectual content.

Elizabeth Holdsworth: Acquisition and interpretation of data for the manuscript and critically revised the manuscript for intellectual content.

Theresa Hunter: Conception, design, and 
interpretation of data for the manuscript and critically revised the manuscript for intellectual content.

All authors participated sufficiently in the work to agree to be accountable for all aspects of the work in ensuring that questions related to the accuracy or integrity of any part of the work are appropriately investigated and resolved.

Prior Presentation. This manuscript is based on work that has been previously presented at the International Society for Pharmacoeconomics and Outcomes Research (ISPOR) virtual conference, May 18-20, 2020.

Medical Writing and Editorial Assistance. The authors would like to thank Gary Sidgwick, PhD, and Katerina Doslikova, PhD, both employees of Adelphi Real World, for medical writing and editorial support in preparing this manuscript.

Disclosures. James Pike, James Piercy, Nicola Booth, and Elizabeth Holdsworth are employees of Adelphi Real World.

Yan Dong and Theresa Hunter are employees of Eli Lilly and Co.

Compliance with Ethics Guidelines. Ethical approval was sought and granted through Western Institutional Review Board (WIRBStudy ID number 1183030). The non-interventional, observational nature of the data collection did not result in patients being placed at risk from the study. Physicians and patients provided informed consent to participate in the study and did not provide any personally identifiable information. All responses were anonymized to preserve respondent (physician and patient) confidentiality and all participating physicians and patients were assigned a study number to aid anonymous data collection and allow linkage of data during data collection and analysis. This study was conducted in accordance with the standards of the Declaration of Helsinki and its later amendments, as well as other regional guidelines and regulations where appropriate. We thank the participants for their involvement in the study.
Data Availability. All data that support the findings of this study are the intellectual property of Adelphi Real World, and thus not publicly available. All requests for access should be addressed directly to Nicola Booth at nicola.booth@adelphigroup.com.

Open Access. This article is licensed under a Creative Commons Attribution-NonCommercial 4.0 International License, which permits any non-commercial use, sharing, adaptation, distribution and reproduction in any medium or format, as long as you give appropriate credit to the original author(s) and the source, provide a link to the Creative Commons licence, and indicate if changes were made. The images or other third party material in this article are included in the article's Creative Commons licence, unless indicated otherwise in a credit line to the material. If material is not included in the article's Creative Commons licence and your intended use is not permitted by statutory regulation or exceeds the permitted use, you will need to obtain permission directly from the copyright holder. To view a copy of this licence, visit http:// creativecommons.org/licenses/by-nc/4.0/.

\section{REFERENCES}

1. Dubash S, McGonagle D, Marzo-Ortega H. New advances in the understanding and treatment of axial spondyloarthritis: from chance to choice. Ther Adv Chronic Dis. 2018;9(3):77-87.

2. Rudwaleit M, van der Heijde D, Landewe R, et al. The development of Assessment of SpondyloArthritis international Society classification criteria for axial spondyloarthritis (part II): validation and final selection. Ann Rheum Dis. 2009;68(6): 777-83.

3. Sieper J, Hu X, Black CM, Grootscholten K, van den Broek RWM, Kachroo S. Systematic review of clinical, humanistic, and economic outcome comparisons between radiographic and non-radiographic axial spondyloarthritis. Semin Arthritis Rheum. 2017;46(6):746-53.

4. Proft F, Poddubnyy D. Ankylosing spondylitis and axial spondyloarthritis: recent insights and impact 
of new classification criteria. Ther Adv Musculoskelet Dis. 2018;10(5-6):129-39.

5. Rudwaleit M, Haibel H, Baraliakos X, et al. The early disease stage in axial spondylarthritis: results from the German Spondyloarthritis Inception Cohort. Arthritis Rheum. 2009;60(3):717-27.

6. Kiltz U, Baraliakos X, Karakostas P, et al. Do patients with non-radiographic axial spondylarthritis differ from patients with ankylosing spondylitis? Arthritis Care Res. 2012;64(9):1415-22.

7. Ciurea A, Scherer A, Exer P, et al. Tumor necrosis factor alpha inhibition in radiographic and nonradiographic axial spondyloarthritis: results from a large observational cohort. Arthritis Rheum. 2013;65(12):3096-106.

8. Wallis D, Haroon N, Ayearst R, Carty A, Inman RD. Ankylosing spondylitis and nonradiographic axial spondyloarthritis: part of a common spectrum or distinct diseases? J Rheumatol. 2013;40(12): 2038-41.

9. Ware JE Jr, Sherbourne CD. The MOS 36-item shortform health survey (SF-36). I. Conceptual framework and item selection. Med Care. 1992;30(6): 473-83.

10. McHorney CA, Ware JE Jr, Raczek AE. The MOS 36-item short-form health survey (SF-36): II. Psychometric and clinical tests of validity in measuring physical and mental health constructs. Med Care. 1993;31(3):247-63.

11. Doward LC, Spoorenberg A, Cook SA, et al. Development of the ASQoL: a quality of life instrument specific to ankylosing spondylitis. Ann Rheum Dis. 2003;62(1):20-6.

12. Kiltz U, van der Heijde D, Boonen A, Braun J. The ASAS Health Index (ASAS HI) - a new tool to assess the health status of patients with spondyloarthritis. Clin Exp Rheumatol. 2014;32(5):105-8.

13. Kiltz U, van der Heijde D, Boonen A, et al. Development of a health index in patients with ankylosing spondylitis (ASAS HI): final result of a global initiative based on the ICF guided by ASAS. Ann Rheum Dis. 2015;74(5):830-5.

14. Powers JH 3rd, Patrick DL, Walton MK, et al. Clinician-reported outcome assessments of treatment benefit: report of the ISPOR clinical outcome assessment emerging good practices task force. Value Health. 2017;20(1):2-14.

15. Kiltz U, van der Heijde D, Boonen A, et al. Measurement properties of the ASAS Health Index: results of a global study in patients with axial and peripheral spondyloarthritis. Ann Rheum Dis. 2018;77(9):1311-7.

16. Anderson P, Benford M, Harris N, Karavali M, Piercy J. Real-world physician and patient behaviour across countries: Disease-Specific Programmes - a means to understand. Curr Med Res Opin. 2008;24(11):3063-72.

17. Babineaux SM, Curtis B, Holbrook T, Milligan G, Piercy J. Evidence for validity of a national physician and patient-reported, cross-sectional survey in China and UK: the Disease Specific Programme. BMJ Open. 2016;6(8):e010352.

18. Ankylosing Spondylitis Quality of Life (ASQoL) questionnaire. http://oml.eular.org/sysModules/ obxOml/docs/ID_32/ASQoL\%20Questionnaire\% 20English.pdf. Accessed 23 Dec 2020.

19. Packham JC, Jordan KP, Haywood KL, Garratt AM, Healey EL. Evaluation of Ankylosing Spondylitis Quality of Life questionnaire: responsiveness of a new patient-reported outcome measure. Rheumatology. 2012;51(4):707-14.

20. Davis JC Jr, Revicki D, van der Heijde DM, et al. Health-related quality of life outcomes in patients with active ankylosing spondylitis treated with adalimumab: results from a randomized controlled study. Arthritis Rheum. 2007;57(6):1050-7.

21. Assessment of SpondyloArthritis international Society Health Index (ASAS-HI) questionnaire. https://www.asas-group.org/clinical-instruments/ asas-health-index/. Accessed 23 Dec 2020.

22. Hastie T, Tibshirani R, Friedman J. The elements of statistical learning: data mining, inference, and prediction. The elements of statistical learning. 2nd ed. New York: Springer-Verlag; 2009.

23. Picard RR, Cook RD. Cross-validation of regression models. J Am Stat Assoc. 1984;79(387):575-83.

24. McCullagh P, Nelder JA. Generalized linear models. 2nd ed. London: Chapman \& Hall/CRC; 1989.

25. Papke E, Woolridge JM. Econometric methods for fractional response variables with an application to 401(k) plan participation rates. J Appl Econ. 1996;11(6):619-32.

26. Fan J, Gijbels I. Local polynomial modelling and its applications. London: Chapman and Hall/CRC; 1996.

27. Barnston A. Correspondence among the correlation [root mean square error] and Heidke verification measures; refinement of the Heidke score notes and correspondence, Climate Analysis Center 1992. http://www.swpc.noaa.gov/sites/default/files/ 
images/u30/Barnston,\%20Anthony\%20G.,\% 201992.pdf. Accessed 23 Dec 2020.

28. Di Carlo M, Lato V, Carotti M, Salaffi F. Clinimetric properties of the ASAS health index in a cohort of Italian patients with axial spondyloarthritis. Health Qual Life Outcomes. 2016;14:78.

29. Kwan YH, Fong W, Tan VIC, et al. A systematic review of quality-of-life domains and items relevant to patients with spondyloarthritis. Semin Arthritis Rheum. 2017;47(2):175-82.

30. Min HK, Lee J, Ju JH, Park SH, Kwok SK. Predictors of Assessment of Spondyloarthritis International Society (ASAS) Health Index in axial spondyloarthritis and comparison of ASAS health index between ankylosing spondylitis and nonradiographic axial spondyloarthritis: data from the Catholic Axial Spondyloarthritis COhort (CASCO). J Clin Med. 2019;8(4):467. 